(C) 2002 IEEE. Reprinted, with permission, from Kokou Dossou, Numerical analysis of the contribution of the transverse asymmetry in the photo-induced index change profile to the birefringence of optical fiber . Lightwave Technology, Journal of (Volume:20 , Issue: 8 ), Aug 2002. This material is posted here with permission of the IEEE. Such permission of the IEEE does not in any way imply IEEE endorsement of any of the University of Technology, Sydney's products or services. Internal or personal use of this material is permitted. However, permission to reprint/republish this material for advertising or promotional purposes or for creating new collective works for resale or redistribution must be obtained from the IEEE by writing to pubs-permissions@ieee.org. By choosing to view this document, you agree to all provisions of the copyright laws protecting it 


\title{
Numerical analysis of the contribution of the transverse asymmetry in the photo-induced index change profile to the birefringence of optical fiber
}

\author{
K. Dossou ${ }^{(1,2)}$, Member, OSA, S. LaRochelle ${ }^{(2)}$, Member, IEEE, Member, \\ OSA, and M. Fontaine ${ }^{(1)}$, Member, OSA \\ Canadian Institute for Photonic Innovations
}

(1) Département d'informatique

Université du Québec à Hull, Québec, Canada J8X 3X7

marie_fontaine@uqah.uquebec.ca

(2) Canada Research Chair in Optical Fibre Communications and Components

Département de Génie électrique et de génie informatique

Centre d'optique, photonique et lasers (COPL)

Université Laval, Québec, Canada G1K 7P4

larochel@gel.ulaval.ca

\begin{abstract}
The birefringence of an optical fiber resulting from an asymmetry of the index profile is numerically evaluated using a finite element method with a full-vectorial formulation. Such asymmetric index profiles, assumed to vary exponentially across the fiber core, could be induced during the writing of fiber Bragg gratings with UV sideexposure techniques. The results reveal that the birefringence is a quadratic function of the effective index change. An asymmetry coefficient near $0.4 \mu \mathrm{m}^{-1}$ maximizes the birefringence. The calculated photo-induced birefringence is negligible if the index change is lower than $5 \times 10^{-4}$. However, the birefringence can reach $5 \times 10^{-6}$ for large values of index change. The numerical method presented could be applied to the modeling of other asymmetric index profiles.
\end{abstract}

Index Terms: waveguide birefringence, photo-induced birefringence, asymmetric index profile, fiber grating, Bragg gratings, finite element analysis, vectorial formulation. 


\section{INTRODUCTION}

Penalties associated to polarization mode dispersion (PMD) become increasingly important at high bit rate, for example, systems transmitting at $40 \mathrm{GBit} / \mathrm{s}$ can typically tolerate PMD of only a few ps. In this context, it is important to minimize the contribution of optical fiber components to the total PMD of the fiber link. The differential group delay (DGD) of photo-induced gratings is related to the fiber birefringence. In the case of chirped gratings used for dispersion compensation, a fiber birefringence as low as $10^{-6}$ can result in an average DGD of several ps [1-2]. In these gratings, it is also likely that polarization dependent loss (PDL) will be coupled to the PMD at some wavelengths because the birefringence will cause the different polarization modes to interact with different parts or the grating. In other applications, the fiber birefringence can be beneficial, for example, to allow the operation of fiber laser devices in a single polarization or to generate a beat signal from the two orthogonal polarized modes [3-5]. In all cases, a photo-induced component can be added to the initial birefringence of the pristine fiber during the grating writing process. In the early days of the development of fiber Bragg gratings, the anisotropy of the photo-induced index change was studied in the context of the realization of polarization rocking filters [6-7]. More recently, the origin of the photo-induced birefringence was investigated in order to improve the performance of components developed for telecommunication applications [8-9].

Previous studies have identified two contributions to the photo-induced birefringence of gratings: the orientation of the writing beam polarization and the asymmetry of the 
index change in the transverse plane [8-9]. The former contribution is attributed to preferential bleaching of defects having their dipole moments aligned with the polarization of the writing UV beam. This anisotropy of the photo-induced index change is observed, for example, during the writing of rocking filters with visible light propagating in the core of a birefringent fiber [7]. In the case of external UV writing, Erdogan and Mizrahi showed that the photo-induced birefringence is reduced by orienting the UV polarization in the incidence plane, almost along the optical fiber axis (p-polarization). Depending on the optical fiber type and exposure conditions, the birefringence was then reduced by factors of 3 to 10 in comparison to s-polarization writing [8]. To explain the remaining birefringence, the transverse asymmetry of the index profile, as well as a small contribution from the UV light still polarized in the transverse plane, must be considered. The contribution from the transverse asymmetry was investigated and reduced by Vengsarkar et al using double exposure of the fiber [9]. After a first exposure, the optical fiber was rotated by 180 and exposed on the other side to obtain a more uniform index change over the fiber core cross-section. A reduction of the birefringence by more than $75 \%$ was observed with the double exposure technique under both p- and s-polarization writing condition. This result indicated that the transverse asymmetry of the index profile could have a major impact on the photoinduced birefringence.

In the study of Vengsarkar et al., the ratio of the photo-induced birefringence was only reduced by a factor of two when changing the polarization of the writing beam from s- to p-polarization. Erdogan and Mizrahi showed a reduction by a factor of 6 for a similar hydrogen-loaded standard communication fiber under similar exposure 
conditions. The importance of the photo-induced birefringence caused by the asymmetry of the transverse profile index thus seemed very sensitive to exposure conditions. Erdogan and Mizrahi also observed that the amount of remaining birefringence varied with the optical fiber type, with no direct correlation to the photosensitivity or the Germania content.

In this study, we numerically investigate the contribution of the transverse asymmetry of the index change profile to the photo-induced birefringence. To describe the transverse asymmetry of the index profile, we use a simple model that is presented in Section 2. It is based on an exponential variation of the index change across the fiber core. Saturation of the index change near the illuminated side is also considered. To compute the photo-induced birefringence, a numerical method based on a finite-element analysis is used to determine the fiber modes and their propagation constants for different asymmetric index profiles. The numerical method based on a full vectorial formulation is introduced in Section 3. Section 4 presents the numerical results and investigates the asymmetry profiles that lead to the largest birefringence. Discussions of the results and conclusion follow in the next sections.

\section{MODELING OF THE TRANSVERSE PROFILE}

To precisely model the transverse profile of the index change, the exact phenomena at the origin of the photosensitive response would need to be known in detail. For example, a model based on the bleaching of defects by one-photon absorption was investigated to describe the growth of fiber Bragg gratings [10]. However, rather than measuring an 
exponential growth of the index change, it was found that the modification of the index change was better described by a power law $\Delta \mathrm{n}=\mathrm{Ct}^{\mathrm{b}}$ with $\mathrm{b} \cong 0.3$ and a proportionality factor, C, function of the writing intensity [10]. The saturated index change also depends on intensity and, at a given time, this index change can be described by a similar power law $\Delta \mathrm{n}=\mathrm{DI}^{\mathrm{f}}$ with, in this case, $\mathrm{f} \cong 0.5$ and a proportionality factor, $\mathrm{D}$, that varies with time. The discrepancy between the one-photon absorption model and the actual growth of the index change is not surprising considering that other mechanisms, such as stress relief and densification, can contribute to the resulting index change. An expression describing the growth of the index change as a function of time and intensity is therefore not available at this time. Furthermore, to determine the intensity across the fiber core, the model would also have to include the local bleaching of the absorption band as a function of time and intensity. The focalisation of the incident UV gaussian beam by the aircladding interface would also have to be considered to establish the distribution of intensity incident on the core. In the absence of a complete model, we have therefore chosen to investigate the impact of an asymmetric transverse profile using a simple function to describe the variation of the index change across the core.

The index change is assumed to follow a decreasing exponential function across the fiber core with possible saturation of the index change over a region of length $\delta$ on the illuminated side. We choose here a coordinate system where the Z-axis coincides with the longitudinal axis of the fiber, the X-Y plane with the transverse section of the fiber and the origin of the system with the center of the fiber core. The transverse index change profile across the core is then 


$$
\Delta n(x, y)=\left\{\begin{array}{cc}
A \alpha & \text { for } \ell(x, y) \leq \delta \\
A \alpha \exp [-\alpha(\ell(x, y)-\delta)] & \text { for } \ell(x, y) \geq \delta
\end{array},\right.
$$

where the parameter $\alpha$ determines the asymmetry of the index profile and the product A $\alpha$ is the maximum photo-induced index change at the fiber core boundary. The function $\ell(\mathrm{x}, \mathrm{y})$ represents the distance of a given point from the illuminated side of the core, e.g. $\ell(x, y)=x+\sqrt{\rho^{2}-y^{2}}$ for illumination along the $x$-axis, with $x^{2}+y^{2} \leq \rho^{2}$ and $\rho$ is the fiber core radius. Without saturation of the index change and neglecting bleaching of the absorption band, equation (1) would represent an index change proportional to the absorbed intensity where A would be related to the photosensitive response characteristic of the material. In germanium doped silica fiber, the absorption coefficient of the $240 \mathrm{~nm}$ band is typically of several hundreds $\mathrm{dB} / \mathrm{mm}$ and even higher in hydrogen loaded fiber [11]. For example, absorption of $200 \mathrm{~dB} / \mathrm{mm}$ corresponds to an absorption coefficient of $0.046 \mu^{-1}$. Although the parameter $\alpha$ may be closely related to the absorption coefficient, for the above mentioned considerations related to the complexity of the modeling of the photosensitive response, we prefer to refer to the parameter $\alpha$ as the asymmetry coefficient.

Vengsarkar et al. measured the transverse refractive index profile of a fiber exposed to UV radiation [9]. The index profile was measured along the x-axis of the fiber core after exposure to UV radiation. To fit the proposed expression to the profile presented in [9], the index profile of the pristine fiber would have to be known and it should be subtracted from the index profile to obtain the index change. We do not know the precise shape of the index profile, but the author specify that the fiber was a hydrogen 
loaded standard telecom fiber with $\Delta=0.3 \%$. Therefore, after subtracting a uniform step index profile from the data presented in [9], a fit of (1) to the transverse profile of the

index change gives an asymmetry coefficient $\alpha=0.2 \mu \mathrm{m}^{-1}$, a maximum index change at the core-cladding interface of $A \alpha=0.0067$ and a saturation length of $0.5 \mu \mathrm{m}$. Figure 1 shows an example of a transverse index profile at $\mathrm{y}=0$ for $\mathrm{A} \alpha=0.005$ and $\alpha=0.2 \mu \mathrm{m}^{-1}$ with and without saturation.

\section{NUMERICAL METHOD AND MODAL ANALYSIS}

Different numerical methods could be used to solve Maxwell's equations in the frequency domain for analyzing the modal properties of waves propagating in optical waveguides. Among them, the finite-element method (FEM) is one of the most powerful and flexible numerical tools because it enables one to analyze any arbitrarily shaped waveguide, including asymmetrical shape as discussed previously. For investigating correctly polarization effects like birefringence and PMD, a full vectorial approach is preferable over conventional scalar approaches in which polarization effects are included as a perturbation. Various full-vectorial formulations of the FEM exist including the vectorial $\vec{E}$ - and $\vec{H}$-field formulations [12] and the mixed $\left(E_{z}, H_{z}\right)$ formulation [13]. Although the last one has been proved to be successful for evaluating precisely PMD and modal chromatic dispersion in highly birefringent fibers, the advantage of using the vectorial field formulations is that it reduces the Maxwell's equation to a generalized eigenvalue problem. In this paper, a vectorial field formulation is used. 


\section{A. Vectorial Field Formulation}

Depending whether the $\vec{E}$ - or $\vec{H}$-field formulation is used, solving the Maxwell's equation is equivalent to solving the vectorial partial differential equation

$$
\nabla \times(\mathrm{p} \nabla \times \vec{\varphi})-\mathrm{k}_{0}^{2} \mathrm{q} \vec{\varphi}=0
$$

where $p=1$ and $q=n^{2}(x, y, z)$ when $\vec{\varphi}$ is the electric field $\vec{E}$ while $p=1 / n^{2}(x, y, z)$ and $q=1$ when $\vec{\varphi}$ is the magnetic field $\vec{H}$. Here thermal stress and absorption effects are disregarded so it is assumed that the optical waveguide materials are isotropic and nonabsorbent with a real scalar refractive index distribution $n(x, y, z)$. As usual, $\mathrm{k}_{0}=2 \pi / \lambda_{0}$ represents the wave number where $\lambda_{0}$ is the wavelength in the vacuum. To simplify the discussion text, we assume the electric field formulation for the remaining of this paper. Similar results are obtained by using the $\vec{H}$-field formulation.

\section{B. Variational formulation}

In this paper, because of the assumptions on the dielectric material, we can assume that the field components $E_{x}, E_{y}, E_{z}$ of each propagating mode

$$
\vec{E}(x, y, z)=\left\{\begin{array}{c}
E_{x}(x, y) \\
E_{y}(x, y) \\
j E_{z}(x, y)
\end{array}\right\} e^{j \beta z}
$$

take real value only [14, Chap 3, section 30-4] and, consequently, complex valued computation can be avoided. Taking this type of z-dependence into account by 
substituting (3) into equation (2), one is lead to the following partial differential equations spectral problem

$$
\left\{\begin{array}{c}
\nabla \times\left(\left(\nabla_{t} \times E_{t}\right) \vec{Z}\right)-\beta \nabla E_{z}+\left(\beta^{2}-n^{2} k_{0}^{2}\right) E_{t}=0 \\
\beta \operatorname{div}\left(E_{t}\right)-\operatorname{div}\left(\nabla E_{z}\right)-n^{2} k_{0}^{2} E_{z}=0
\end{array}\right.
$$

where $\nabla_{t}$ denotes the transverse gradient so that $\nabla_{t} \times E_{t}=\frac{\partial E_{y}}{\partial x}-\frac{\partial E_{x}}{\partial y}$.

When a FEM is used to solve a partial differential equation, this one must be rewritten in an equivalent variational formulation before being discretized. We will derive the variational problem (weak problem) from (4) in a straightforward manner. For this, we shall assume that we work in appropriate functional spaces. Taking the standard Hilbert's space inner product of the left-hand side of (4) with any test functions

$$
\vec{F}=\left\{\begin{array}{l}
F_{t} \\
F_{z}
\end{array}\right\}
$$

and transforming the result by integration by parts, one is lead to

$$
\left\{\begin{array}{l}
\beta^{2} \iint_{\Omega} E_{t} \cdot F_{t} d x d y-\beta \iint_{\Omega}\left(\nabla E_{z}\right) \cdot F_{t} d x d y \\
+\iint_{\Omega}\left(\left(\nabla_{t} \times E_{t}\right)\left(\nabla_{t} \times F_{t}\right)-n^{2} k_{0}^{2} E_{t} \cdot F_{t}\right) d x d y+\int_{\partial \Omega}\left(\nabla_{t} \times E_{t}\right)\left(F_{t} \cdot \vec{\tau}\right) d s=0 \\
\iint_{\Omega}\left(\left(\nabla E_{z}\right) \cdot\left(\nabla F_{z}\right)-n^{2} k_{0}^{2} E_{z} F_{z}\right) d x d y-\beta \iint_{\Omega} E_{t} \cdot\left(\nabla F_{z}\right) d x d y \\
+\int_{\partial \Omega} F_{z}\left(\beta E_{t}-\nabla E_{z}\right) \cdot \vec{v} d s=0
\end{array}\right.
$$

where $\partial \Omega$ is the boundary of $\Omega, \Omega$ being the transverse section of the fiber. The vectors

$$
\vec{v}=\left\{\begin{array}{c}
v_{x} \\
v_{y} \\
0
\end{array}\right\} \text { and } \vec{\tau}=\left\{\begin{array}{c}
-v_{y} \\
v_{x} \\
0
\end{array}\right\}
$$


denote respectively the normal outward vector to $\partial \Omega$ and an unitary tangential vector to $\partial \Omega$.

Assuming electric wall boundary condition $\left\{\begin{array}{l}F_{t} \\ F_{z}\end{array}\right\} \times \vec{\tau}=0$, i.e., $F_{t} \cdot \vec{\tau}=0$ and $F_{z}=0$ on $\partial \Omega$, cancellation of the line integrals in (6) occurs. Finally the propagating mode problem corresponds to finding $\beta \in R,\left(E_{t}, E_{z}\right)$ such that $\left(E_{t}, E_{z}\right) \neq(0,0)$ and $\forall\left(\mathrm{F}_{\mathrm{t}}, \mathrm{F}_{\mathrm{z}}\right)$, from

$$
\left\{\begin{array}{l}
\beta^{2} \iint_{\Omega} \mathrm{E}_{\mathrm{t}} \cdot \mathrm{F}_{\mathrm{t}} \mathrm{dxdy}-\beta \iint_{\Omega}\left(\nabla \mathrm{E}_{\mathrm{z}}\right) \cdot \mathrm{F}_{\mathrm{t}} \mathrm{dxdy} \\
+\iint_{\Omega}\left(\left(\nabla_{\mathrm{t}} \times \mathrm{E}_{\mathrm{t}}\right)\left(\nabla_{\mathrm{t}} \times \mathrm{F}_{\mathrm{t}}\right)-\mathrm{n}^{2} \mathrm{k}_{0}{ }^{2} \mathrm{E}_{\mathrm{t}} \cdot \mathrm{F}_{\mathrm{t}}\right) \mathrm{dx} \mathrm{dy}=0 \\
\iint_{\Omega}\left(\left(\nabla \mathrm{E}_{\mathrm{z}}\right) \cdot\left(\nabla \mathrm{F}_{\mathrm{z}}\right)-\mathrm{n}^{2} \mathrm{k}_{0}{ }^{2} \mathrm{E}_{\mathrm{z}} \mathrm{F}_{\mathrm{z}}\right) \mathrm{dxdy}-\beta \iint_{\Omega} \mathrm{E}_{\mathrm{t}} \cdot\left(\nabla \mathrm{F}_{\mathrm{z}}\right) \mathrm{dxdy}=0
\end{array} .\right.
$$

If $\beta,\left(E_{t}, E_{z}\right)$ is a solution to problem (8), the fact that the field product of $\left(E_{t}, E_{z}\right)$ with each test function has to be zero, implies that the $\vec{E}$ field residual of problem (4) is also zero. However the residual relative to (4) of the solution to the discretized problem (10) below is only zero in some average sense since the test functions are restricted to the finite dimensional space generated by the basis functions.

\section{Discretization}

The discretization of the surface integrals (8) in the transverse plane of the fiber is performed by using a vector edge finite element [15]. The transverse component of the field is approximated by a linear edge element $P_{1}$ (polynomial of order one) while the longitudinal component is approximated by a quadratic nodal element $P_{2}$ (polynomial of order 2). Edge elements are appropriate because, unlike the standard nodal finite element, 
they do not produce spurious solutions and the boundary conditions at material interfaces are well enforced.

The transverse and the axial components in each element $e$ is expanded as

$$
\vec{E}=\left\{\begin{array}{c}
E_{x} \\
E_{y} \\
j E_{z}
\end{array}\right\}=\left\{\begin{array}{c}
\{U\}^{T}\left\{E_{t}\right\}_{e} \\
\{V\}^{T}\left\{E_{t}\right\}_{e} \\
j\{N\}^{T}\left\{E_{z}\right\}_{e}
\end{array}\right\}
$$

where $\mathrm{T}$ stands for matrix transpose, $\left\{\mathrm{E}_{\mathrm{t}}\right\}_{\mathrm{e}}$ is the edge variable for the transverse component, $\left\{\mathrm{E}_{\mathrm{z}}\right\}_{\mathrm{e}}$ the axial nodal values, $\{\mathrm{U}\},\{\mathrm{V}\}$ contain the $\mathrm{P}_{1}$ polynomial expression for the edge vector basis and $\{\mathrm{N}\}$ describes the $\mathrm{P}_{2}$ polynomial coefficients for the basis of the nodal elements. More details on the shape function polynomial coefficients and the finite element basis can be found in [15].

Application of Galerkin procedure to (8) leads to the quadratic eigenvalue problem

$$
\left\{\begin{array}{l}
\beta^{2}\left[M_{t t}\right]\left\{E_{t}\right\}-\beta\left[K_{t z}\right]\left\{E_{z}\right\}-\left[K_{t t}\right]\left\{E_{t}\right\}=0 \\
{\left[K_{z z}\right]\left\{E_{z}\right\}-\beta\left[K_{z t}\right]\left\{E_{t}\right\}=0}
\end{array}\right.
$$

which is written in matrix form

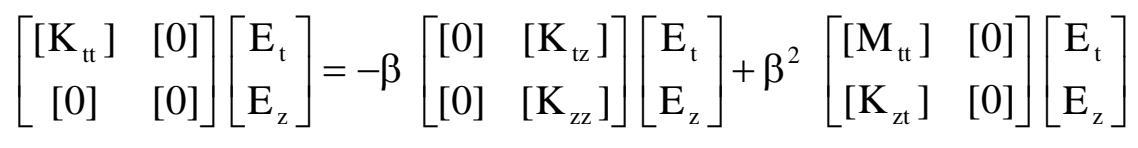


where

$$
\begin{aligned}
& {\left[K_{t t}\right]=\sum_{e} \iint_{\Omega_{e}}\left[n^{2} k_{0}^{2}\{U\}\{U\}^{T}+n^{2} k_{0}^{2}\{V\}\{V\}^{T}-\left\{U_{y}\right\}\left\{U_{y}\right\}^{T}-\left\{V_{x}\right\}\left\{V_{x}\right\}^{T}+\left\{U_{y}\right\}\left\{V_{x}\right\}^{T}\right.} \\
& \left.+\left\{V_{x}\right\}\left\{U_{y}\right\}^{T}\right] d x d y \\
& {\left[K_{t z}\right]=\sum_{e} \iint_{\Omega_{e}}\left[\{U\}\left\{N_{x}\right\}^{T}+\{V\}\left\{N_{y}\right\}^{T}\right] d x d y} \\
& {\left[K_{z t}\right]=\left[K_{t z}\right]^{T}} \\
& {\left[K_{z z}\right]=\sum_{e} \iint_{\Omega_{e}}\left[\left\{N_{x}\right\}\left\{N_{x}\right\}^{T}+\left\{N_{y}\right\}\left\{N_{y}\right\}^{T}-n^{2} k_{0}^{2}\{N\}\{N\}^{T}\right] d x d y} \\
& {\left[M_{t t}\right]=\sum_{e} \iint_{\Omega_{e}}\left[\{U\}\{U\}^{T}+\{V\}\{V\}^{T}\right] d x d y}
\end{aligned}
$$

and where $\left\{N_{x}\right\}=\partial\{N\} / \partial x, \quad\left\{N_{y}\right\}=\partial\{N\} / \partial y, \quad\left\{U_{x}\right\}=\partial\{U\} / \partial x, \quad\left\{\mathrm{U}_{\mathrm{y}}\right\}=\partial\{\mathrm{U}\} / \partial \mathrm{y}$, $\left\{V_{x}\right\}=\partial\{V\} / \partial x$ and $\left\{V_{y}\right\}=\partial\{V\} / \partial y$. The summation $\sum_{e}$ is carried over all the different finite element triangles $\Omega_{e}$. The substitution $\mathrm{E}_{\mathrm{z}}=-\beta \hat{\mathrm{E}}_{\mathrm{z}}$ gives the following generalized eigenvalue problem

$$
\left[\begin{array}{cc}
{\left[K_{t t}\right]} & {[0]} \\
{[0]} & {[0]}
\end{array}\right]\left\{\begin{array}{l}
E_{t} \\
\hat{E}_{z}
\end{array}\right\}=\beta^{2}\left[\begin{array}{ll}
{\left[M_{t t}\right]} & {\left[K_{t z}\right]} \\
{\left[K_{z t}\right]} & {\left[K_{z z}\right]}
\end{array}\right]\left\{\begin{array}{c}
E_{t} \\
\hat{E}_{z}
\end{array}\right\}
$$

It's interesting to point out that problem (12) has the same size as (11) and involves only sparse matrices. We use the ARPACK library [16] to compute numerically the eigenmodes. A shift-and-invert method has been introduced to compute precisely the effective index $\beta / k_{0}$ of the modes polarized along the fast and slow axis of the fiber.

Figures 2A and 2B show typical meshes realized with the software Mefisto [17]. There are 11473 nodes and 5712 elements in Figure 2A as compared to 2089 nodes and 
1032 elements in Figure 2B. Numerical simulations show that the high density of triangles around the interface between the core and the cladding of the fiber, as shown in Fig. 2B, is required to ensure the polarization effects are well taken into account. Simulations also reveal that the use of a number of elements greater than 5712 does not improve significantly the accuracy of the results. So all the numerical results presented in the next section have been realized with the mesh illustrated in Fig. 2A. Note also that the radius of the computational window is eight times larger than the one of the core. In this case, the electric wall condition can be imposed on the solution at the exterior boundary, without producing non-physical reflections since the field decreases exponentially through the boundary.

\section{RESULTS AND DISCUSSION}

We investigate numerically the birefringence properties of a single mode optical fiber with a silica cladding ( $\mathrm{n}_{\text {cladding }}=1.444, \mathrm{n}_{\text {core }}=1.4493$ and $\left.\rho=4.15 \mu \mathrm{m}\right)$. The calculations were performed at the wavelength of $1.53 \mu \mathrm{m}$. The effective index of the modes polarized along the fast and the slow axis were calculated, using the method described in Section 3, as a function of the asymmetry coefficient $\alpha$ and of the maximum index change A $\alpha$. The contour plots presented Fig.3 show the contribution of these two parameters to the change in the effective index for the fast axis corresponding to the mode polarized along the $y$-axis. Similar plots could be obtained for the mode polarized along the slow axis. As expected, a larger asymmetry, $\alpha$, results in a smaller modification of the effective index for the same maximum index change, $\mathrm{A} \alpha$, at the core-cladding interface. Figure 4 shows the graphs of the effective index for the mode polarized along 
the fast axis as a function of the maximum index change for two values of the asymmetry coefficient, $\alpha=0.2 \mu \mathrm{m}^{-1}$ and $\alpha=0.4 \mu \mathrm{m}^{-1}$. Once again, similar curves were obtained for the mode polarized along the slow axis. It can be seen that the effective index, for both polarizations, has a strong linear dependence on the maximum index change with a small quadratic contribution. For example, for $\alpha=0.2 \mu \mathrm{m}^{-1}$, the change in effective index is approximately given by $A \alpha / 2$.

The birefringence resulting from the transverse asymmetry was calculated from the effective indices of the two modes and is displayed in Figure 5 as a function of A $\alpha$ and $\alpha$. For a given maximum index change at the core cladding interface, $A \alpha$, there is a value of the asymmetric coefficient that will maximize the photo-induced birefringence typically near $\alpha=0.3 \mu \mathrm{m}^{-1}$ to $\alpha=0.4 \mu \mathrm{m}^{-1}$. Figure 6 shows the birefringence as a function of the maximum index change for $\alpha=0.2 \mu \mathrm{m}^{-1}$ and $\alpha=0.4 \mu \mathrm{m}^{-1}$. The results clearly show that the birefringence resulting from the asymmetry in the transverse profile has a nonlinear and mostly quadratic dependence on the maximum index change. The effect of the saturation of the index change on the birefringence is displayed in Figure 7. It can be seen that saturation of the index change over a length of $1.5 \mu \mathrm{m}$ will increase the birefringence by $30 \%$.

For the asymmetry of the index profile measured in [9] $\left(\alpha=0.2 \mu \mathrm{m}^{-1}\right.$ and $A \alpha=0.007$ ), our calculations give a birefringence that would be $3 \times 10^{-6}$ to $4 \times 10^{-6}$, depending on the length over which the index change saturates. This value is in reasonable agreement with the range of the photo-induced birefringence measured by Erdogan and Mizrahi after exposure to UV light with p-polarization, typically $4 \times 10^{-6}$ to $5 \times 10^{-6}$ [8]. However, Vengsarkar et al. measured a birefringence reaching $1 \times 10^{-5}$ under 
similar exposure conditions. It is not clear why the birefringence measured in the latter case was so much larger than in the former experiment, reaching half the value of the birefringence measured under exposure to s-polarized light. At this point, our calculations do not seem to lead to such a large value of birefringence caused by the transverse profile alone. However, before a definitive conclusion can be drawn, the exact transverse profile of the photo-induced index change would have to be determined, not an easy task as modifications of stress components could also contribute to the observed index change and to the birefringence.

The calculations show that, while the change in the effective index grows linearly with the index change at the core-cladding interface, $A \alpha$, the dependence of the birefringence resulting from the transverse asymmetry is quadratic. In their paper, Erdogan and Mizrahi suggest that the growth of the birefringence is proportional to the growth of the total index change. Although this statement may be correct when considering the component of the photo-induced birefringence resulting from the polarization of the UV writing beam, our calculation predict that the contribution to the birefringence caused by transverse asymmetry of the index profile, should grow quadratically with the index change. As a consequence, the birefringence resulting from asymmetry in the index profile becomes important $\left(>10^{-7}\right)$ only for very strong effective index change $\left(>10^{-3}\right)$. 


\section{CONCLUSION}

In previous experimental studies, side illumination of optical fibers to UV irradiation has been shown to result in photo-induced birefringence. An asymmetry of the index change in the optical fiber transverse plane was proposed as a possible cause to this increased birefringence. In this study, the birefringence resulting from a transverse asymmetry of the core refractive index profile was investigated numerically. The transverse asymmetry was represented by a simple model based on an exponential decay of the photo-induced index change across the core of a standard step index fiber. This model is characterized by two parameters namely the amplitude of the photo-induced index change at the corecladding interface and the coefficient of the exponential term characterizing the asymmetry of the profile. To evaluate the order of magnitude of the resulting birefringence, the propagation constants of the two orthogonally polarized fundamental modes were found using a full-vectorial finite element method. To achieve the required accuracy in the calculations, a large number of elements were needed, particularly at the core-cladding interface, to precisely evaluate the polarization effects. The main advantage of the finite element method presented here is that it could be easily adapted to the study of other asymmetric index profiles including anisotropy due thermal stress effects.

The numerical results show that the linear dependence of the effective index on the photo-induced index change is almost the same for the two modes polarized along the fast and slow axis. Also, the resulting birefringence displays a quadratic dependence on the amplitude of the photo-induced index change. An asymmetry coefficient near $0.4 \mu^{-}$

${ }^{1}$ was found to maximize the photo-induced birefringence. Saturation of the index change across a small portion of the core can also lead to an increase in the calculated 
birefringence. Although the exact variation of the index change in the transverse plane of a UV exposed fiber can differ from the simulated profile, it is believed that the results give an order of magnitude estimate of the resulting birefringence. More specifically, the calculations indicate that the birefringence from the asymmetry of the transverse profile will be negligible, i.e. smaller than $10^{-7}$, when the effective index change is lower than $5 \times 10^{-4}$. For high effective index change, for example $5 \times 10^{-3}$, the birefringence can reach $5 \times 10^{-6}$ or slightly higher.

The impact of this photo-induced birefringence on the performance of fiber grating components remains to be determined. To adequately calculate the polarization properties of fiber gratings, mode coupling taking place at the boundary between the unexposed and the exposed fiber segments will have to be considered. In most cases, the photo-induced birefringence and the birefringence of the pristine fiber are expected to be of the same order of magnitude. The orientation of the initial birefringent axis of the optical fiber during UV exposure will therefore have an impact on the resulting polarization properties of the gratings.

\section{ACKNOWLEDGMENTS}

This work was supported by the Canadian Institute for Photonic Innovations. The authors are grateful to professor Roger Pierre for stimulating discussions. 


\section{REFERENCES}

1. M. Schiano and G. Zaffiro, "Polarisation mode dispersion in chirped fibre gratings", European Conference on Optical Communications, vol. I, pp 403-404, 1998.

2. E.Ciaramella, E. Riccardi and M. Schiano, "System penalties due to polarization mode dispersion of chirped gratings", European Conference on Optical Communications, vol.I, pp.515-516, 1998.

3. M. Ibsen, E. Rønnekliev,G. J. Cowle, M. O. Berendt, O. Haldeler, M. N. Zervas, and R. I. Laming, "Robust high power $(>20 \mathrm{~mW})$ all-fibre DFB lasers with unidirectional and truly single polarization outputs", Conference on Lasers and Electro-Optics, pp.245-246, 1999.

4. G. A. Ball, G. Meltz and W. W. Morey, "Polarimetric heterodyning Bragg-grating fiber-laser sensor", Opt. Lett., vol. 18, pp.1976-1979, 1993.

5. V. Mizrahi, D. J. DiGiovanni, R. M. Atkins, S. G. Grubb, Y.-K.Park, and J.-M. Delavaux, "Stable single-mode erbium fiber-grating laser for digital communication", J. Lightwave Technol., vol. 11, pp. 2021-2025, 1993.

6. K. O. Hill, F. Bilodeau, B. Malo, and D. C. Johnson, "Birefringent photosensitivity in monomode optical fibre: Application to external writing of rocking filters", Electron. Lett., vol. 27, pp.1548-1550, 1991.

7. P. S.-J. Russell and D. P. Hand, "Rocking filter formation in photosensitive high birefringence optical fibres", Electron. Lett., volé 26, pp. 1846-1848, 1990.

8. T. Erdogan and V. Mizrahi, "Characterization of UV-induced birefringence in photosensitive Ge-doped silica optical fibers", J. Opt. Soc. Am. B., vol. 11, pp. 21002105, 1994.

9. A. M. Vengsarkar, Q. Zhong, D. Inniss, W. A. Reed, P. J. Lemaire, and S. G. Koniski, "Birefringence reduction in side-written photo-induced fiber devices by a dual exposure method", Opt. Lett., vol.19, pp.1260-1263, 1994.

10. H. Patrick and S. L. Gilbert, "Growth of Bragg gratings produced by continuouswave ultraviolet light in optical fiber", Opt. Lett., vol. 18, pp.1484-1486, 1993.

11. R. M. Atkins and V. Mizrahi "Observations of changes in UV absorption bands of singlemode germanosilicate core optical fibres on writing and thermally erasing refractive index gratings", Electron. Lett., vol. 28, pp. 743-1744, 1992.

12. M. Koshiba, S. Maruyama, and K. Hirayama, "Simple and efficient finite-element analysis of microwave and optical waveguides", IEEE Transactions on Microwave Theory and Techniques, vol. 40, pp. 371-377, 1992.

13. M. Fontaine, B. Wu, V.P. Tzolov, W.J. Bock, and W. Urbanczyk, "Theoretical and experimental analysis of thermal stress effects on modal polarization properties of highly birefringent optical fibers", J. Lightwave Technol.,vol. 14, pp. 585-591, 1996.

14. A.W. Snyder and Love, J.D., "Optical Waveguide Theory", Chapman and Hall, New York, 1983

15. M. Koshiba, S. Maruyama, and K. Hirayama, "A vector finite element method with the high-order mixed-interpolation-type triangular elements for optical waveguiding problems", Journal of Lightwave Technology, vol. 12, pp.495-502, 1994.

16. ARPACK see the web site: http://www.caam.rice.edu/software/ARPACK/

17. Mefisto see the web site: http://www.ann.jussieu.fr/ perronne/mefisto.gene.html 


\section{BIOGRAPHIES}

Kokou Dossou graduated from University of Lomé (Togo), Department of Mathematics, in 1991; he received his master and Ph.D. (Mathematics) in 1995 and 2000 from Université Laval, Québec, Canada. He is currently a postdoctoral researcher at Université du Québec à Hull, Québec, Canada. He is engaged in research on numerical analysis of electromagnetic fields and finite element analysis. Dr. Dossou is a Member of OSA.

Sophie LaRochelle received a Bachelor's degree in Engineering Physics from Université Laval, Canada, in 1987; and a Ph.D. degree in Optics from the University of Arizona, USA, in 1992. From 1992 to 1996, she was a Research Scientist at the Defense Research Establishment Valcartier, where she worked on electro-optical systems. She is now a professor at the Department of Electrical and Computer Engineering, Université Laval, where she holds the Canada Research Chair in Optical Fibre Communications and Components. Her current research activities are focused on active and passive fiber optics components for optical communication systems including Bragg gratings, multiwavelength lasers, and amplifiers. Dr. LaRochelle is a member of OSA and of IEEELEOS.

Marie Fontaine received the M.Sc. and Ph.D. degrees in Physics form Université Laval, Canada in 1978 and 1982, respectively. From 1983 to 1985, she was with the Division of Electrical Engineering, National Research Council, Canada, as a Postdoctoral Fellow. She is currently a Professor with the Department of Computer Science at the Université du Québec à Hull, Canada. Her research interests include linear and nonlinear optics. Dr. Fontaine is a Member of OSA. 


\section{FIGURE CAPTIONS}

Figure 1. Graph of the index change profile. A) Two dimensional index change profile with $\alpha=0.2 \mu \mathrm{m}^{-1}$ and $A \alpha=0.005$. B) The index change along the $\mathrm{x}$-axis for $\mathrm{A} \alpha=0.005$ and $\alpha=0.2 \mu \mathrm{m}^{-1}$ without saturation (solid line) and with saturation over a length $\delta=0.5 \mu \mathrm{m}$ (dashed line).

Figure 2. Finite element discretization of the fiber cross-sections with A) 5712 triangles and 11473 nodes and B) 1032 triangles and 2089 nodes.

Figure 3. Effective index of the modes polarized along the fast axis as function of the parameters $\alpha$ and $A \alpha$.

Figure 4. Effective index of the modes polarized along the fast axis as function of $A \alpha$ for $\alpha=0.2 \mu \mathrm{m}^{-1}$ (solid line) and $0.4 \mu \mathrm{m}^{-1}$ (dashed curve).

Figure 5. Contour plots of the photo-induced birefringence as a function of the parameters $A \alpha$ and $\alpha, A)$ for $\alpha$ smaller than $0.2 \mu \mathrm{m}^{-1}$ and B) for $\alpha$ up to $1 \mu \mathrm{m}^{-1}$.

Figure 6. Birefringence as a function of the photosensitive response $A \alpha$ for $\alpha=0.2 \mu \mathrm{m}^{-1}$ (solid line) and $0.4 \mu \mathrm{m}^{-1}$ (dashed curve).

Figure 7. Effect of the saturation of the index change on the birefringence calculated for $\mathrm{A} \alpha=0.005$ and $\alpha=0.2 \mu \mathrm{m}^{-1}$. 


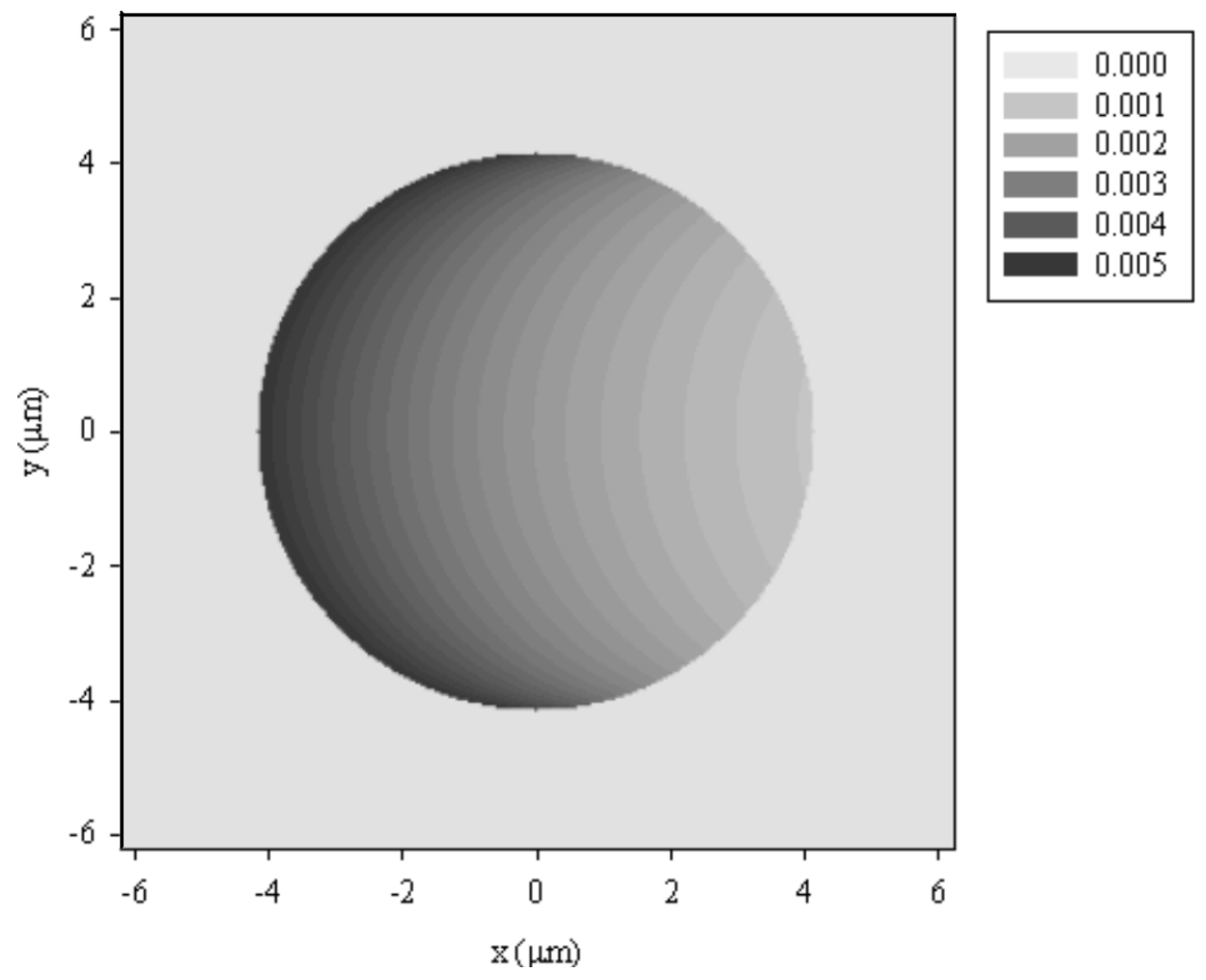

FIG 1A 


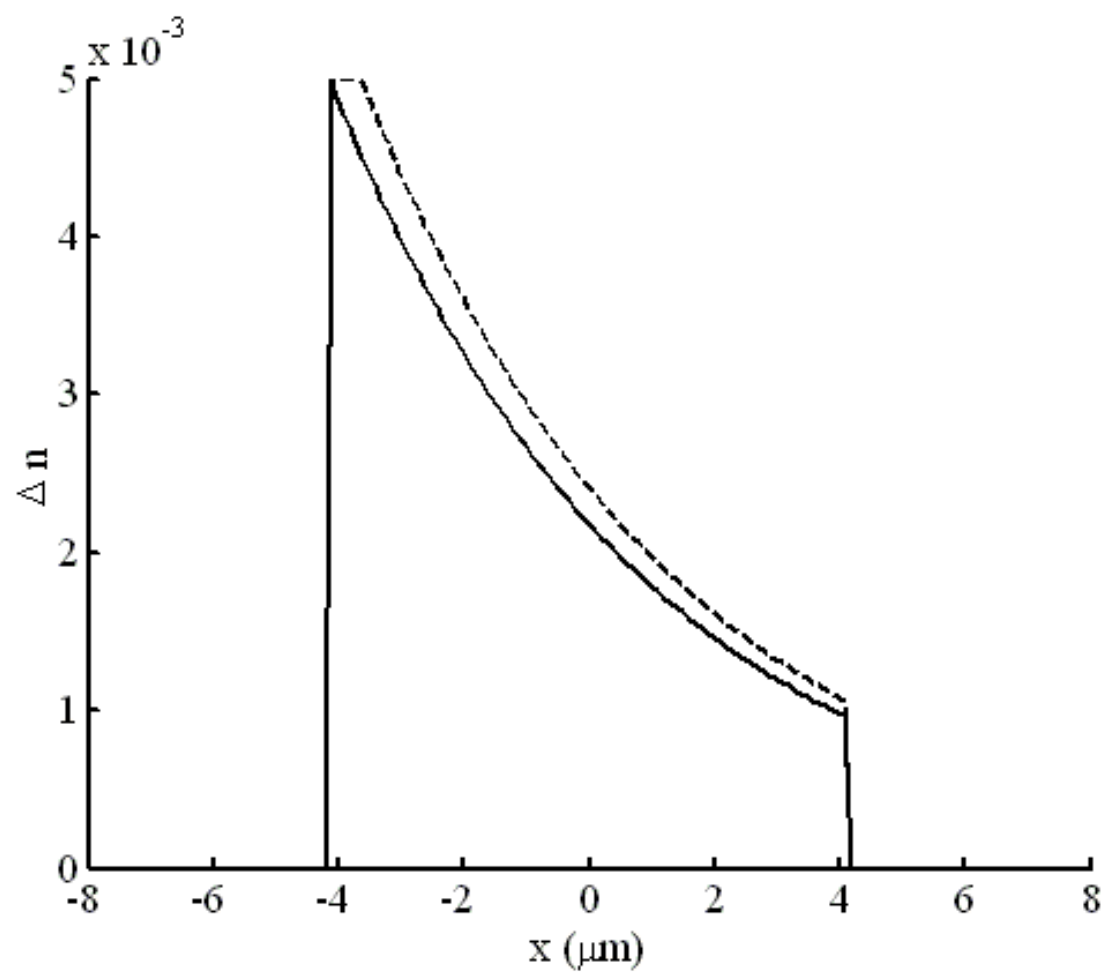

FIG 1B 
Figure supprimée pour réduire la taille du fichier

FIG 2A 
Figure supprimée pour réduire la taille du fichier

FIG 2B 


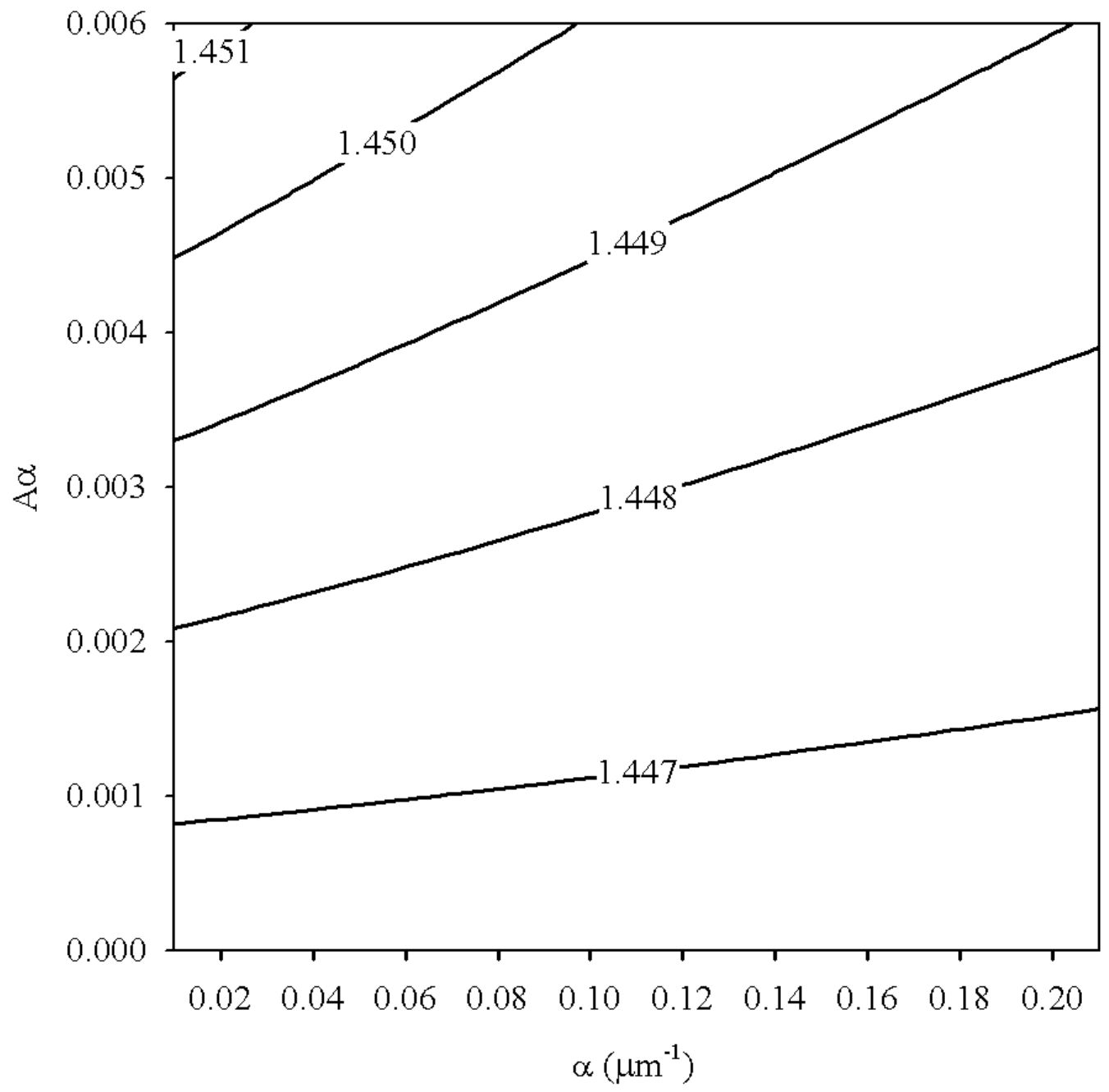

FIG 3 


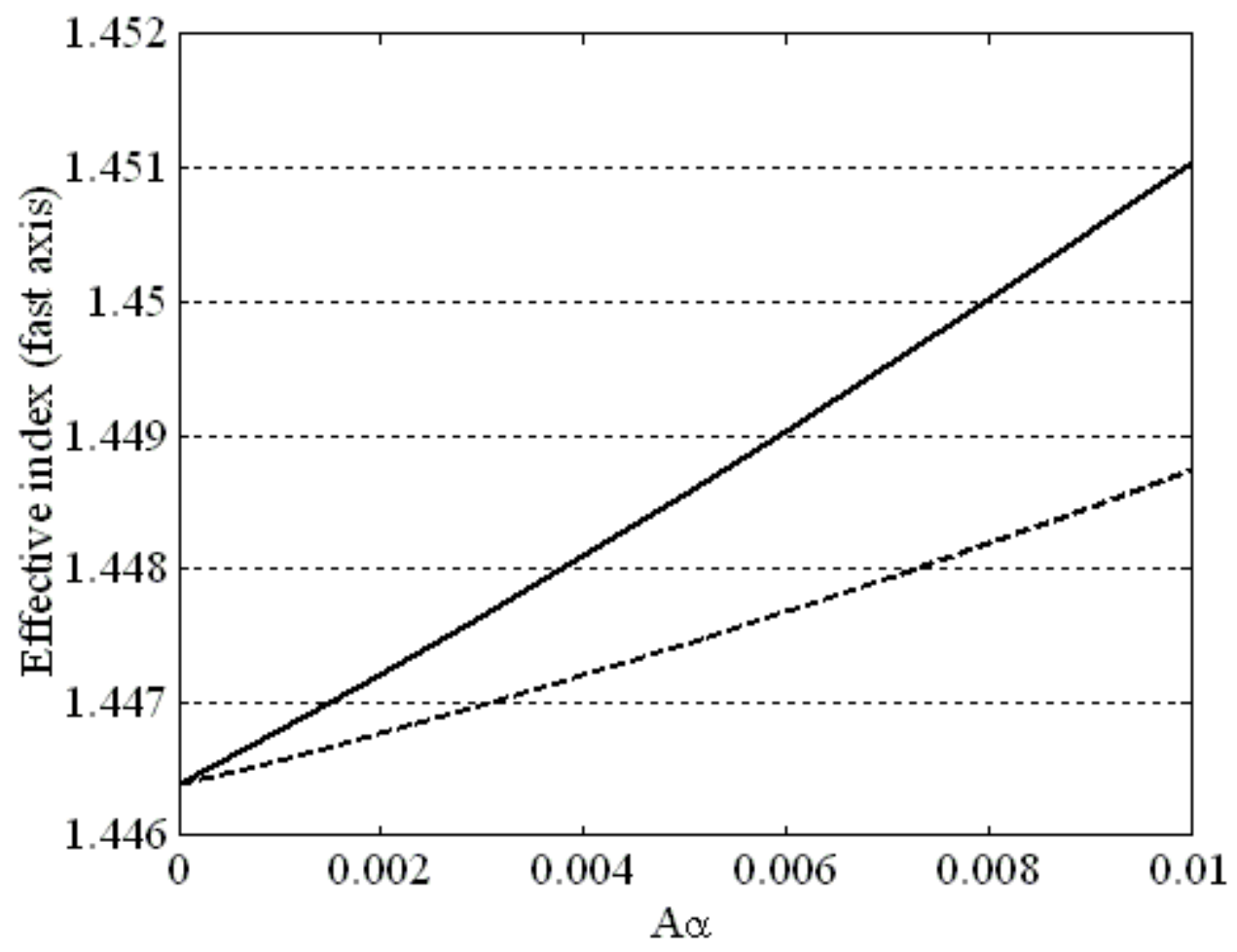

FIG 4 


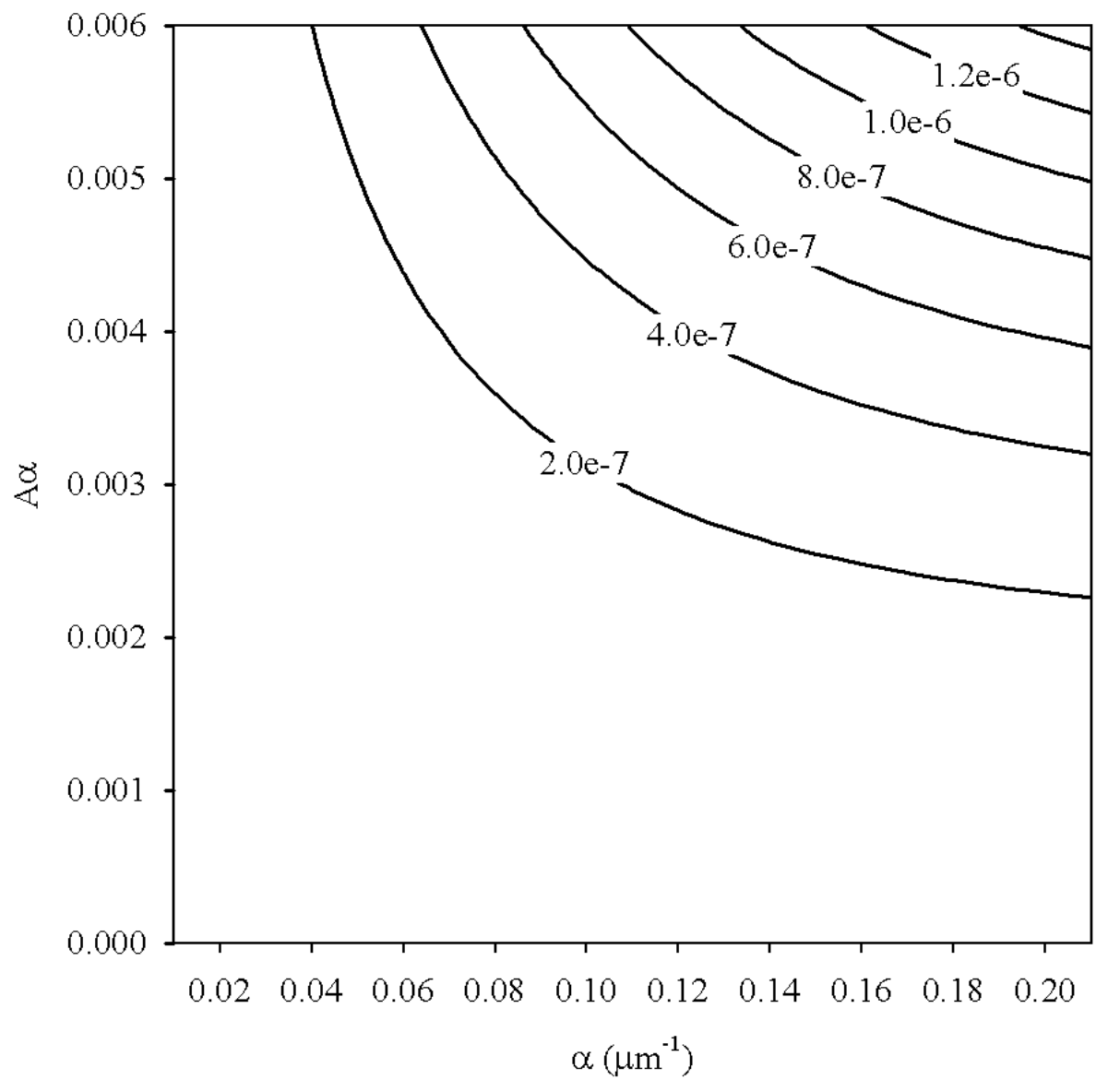

FIG 5A 


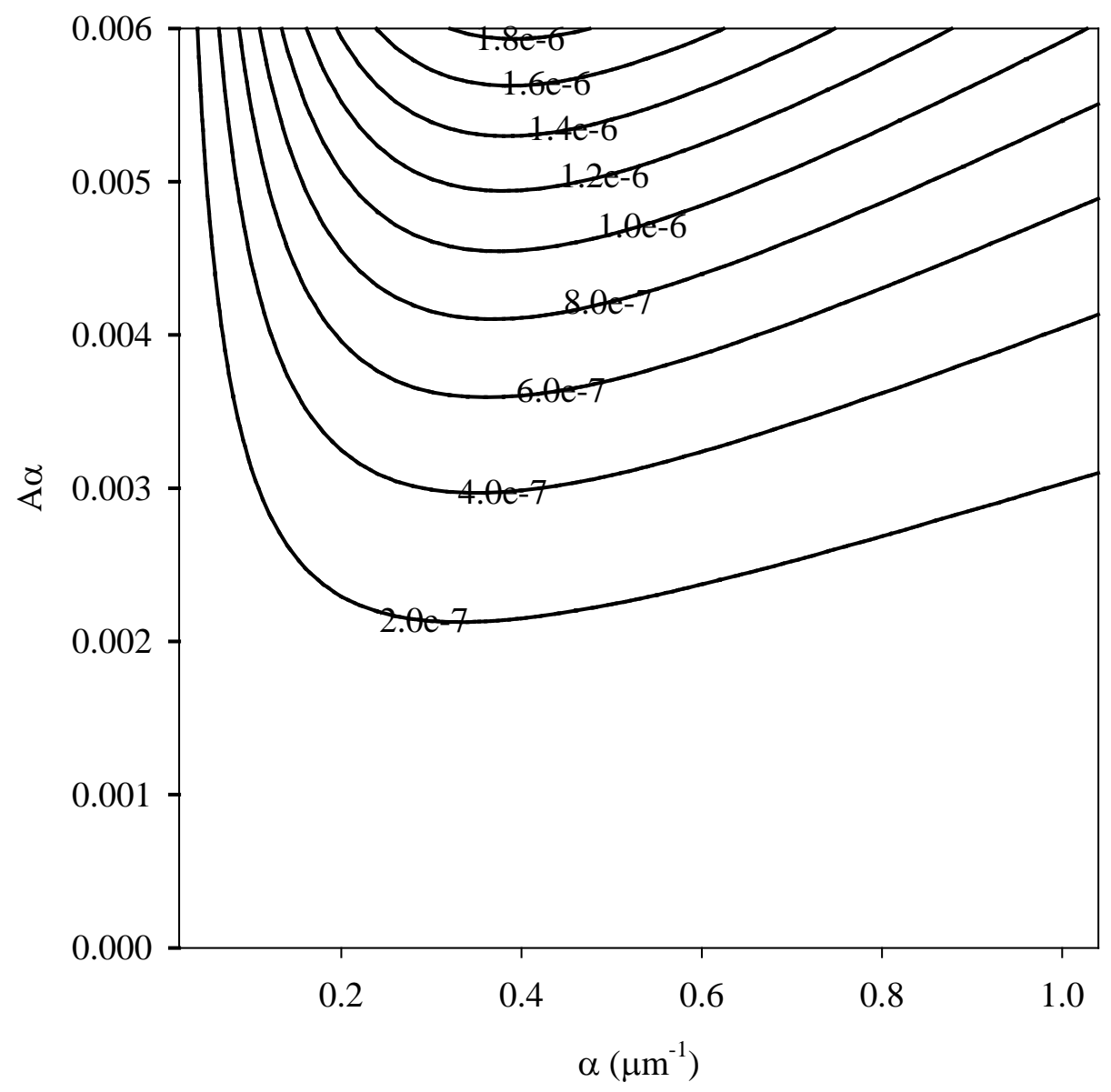

FIG 5B 


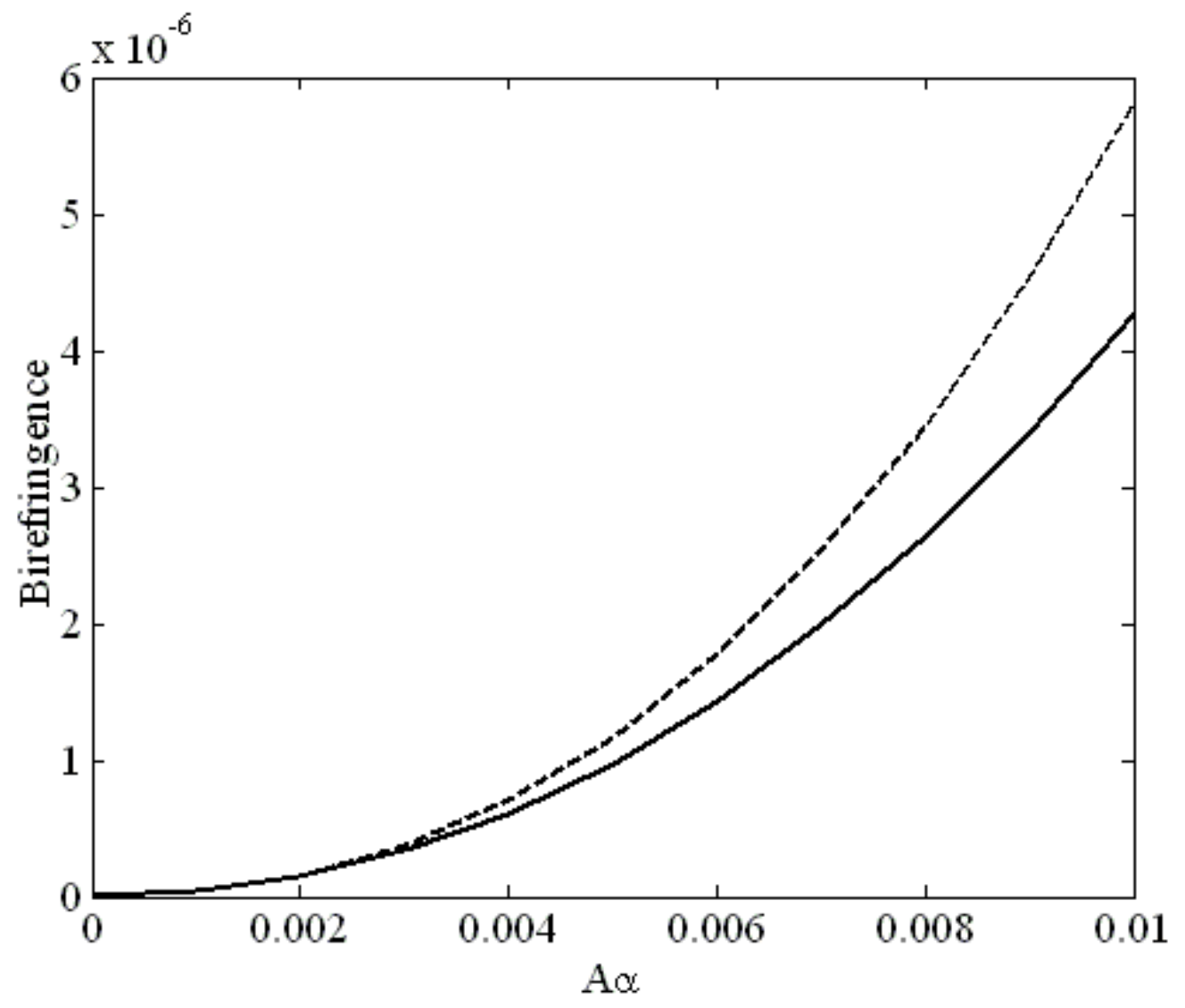

FIG 6 


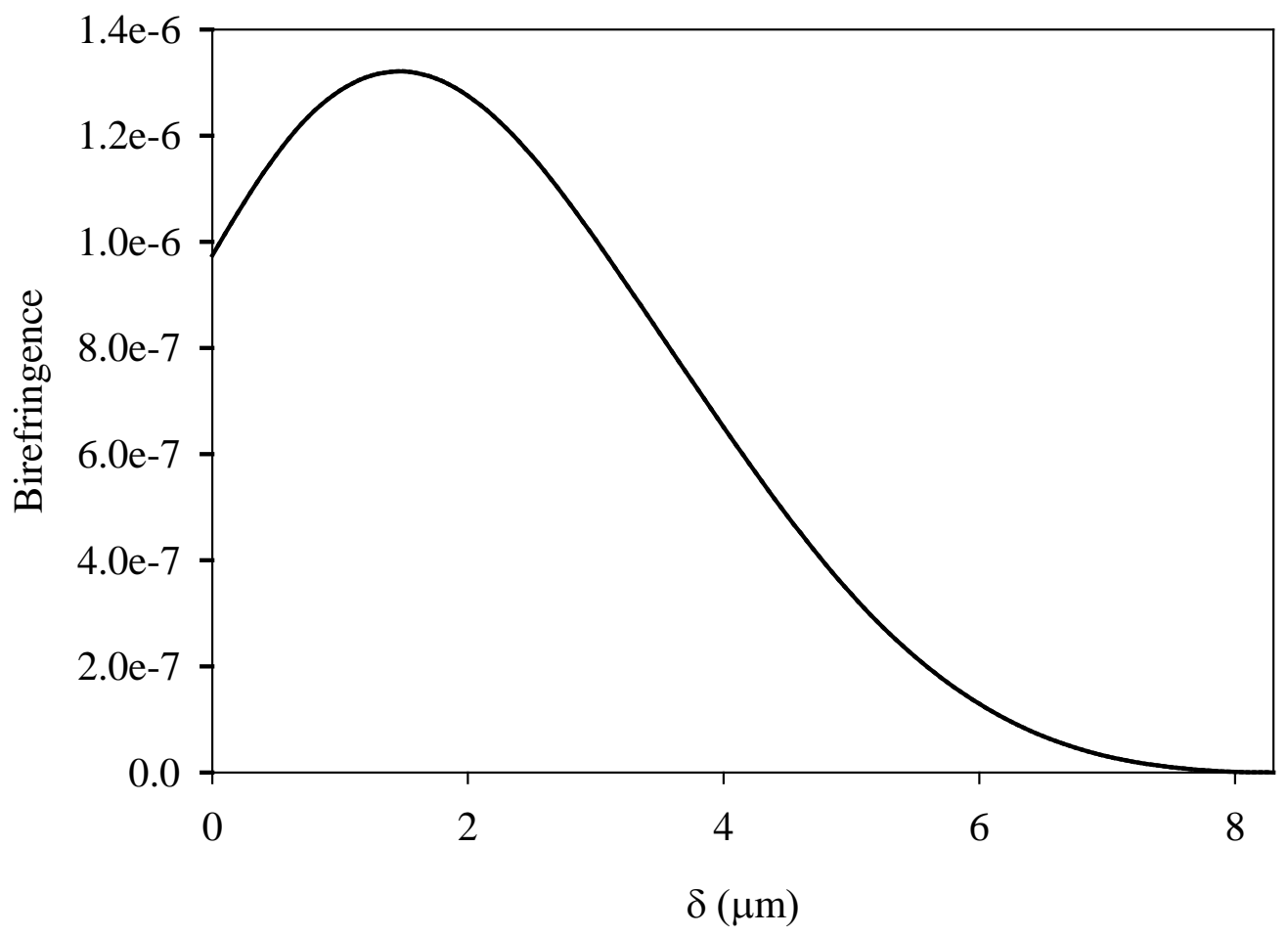

FIG 7 